Article

\title{
Development of Poly(L-Lactic Acid)-Based Bending Actuators
}

\author{
Daniela M. Correia ${ }^{1,2, *(\mathbb{D})}$, Liliana C. Fernandes ${ }^{2}$, Bárbara D.D. Cruz ${ }^{3}$, Gabriela Botelho ${ }^{3}{ }^{\mathbb{D}}$, \\ Verónica de Zea Bermudez ${ }^{1,4, *(D)}$ and Senentxu Lanceros-Méndez ${ }^{5,6}$ (D) \\ 1 CQ-VR, University of Trás-os-Montes e Alto Douro, 5000-801 Vila Real, Portugal \\ 2 Centre of Physics, University of Minho, 4710-057 Braga, Portugal; lilianafernandes1411@gmail.com \\ 3 Centre of Chemistry, University of Minho, 4710-057 Braga, Portugal; barbara.cruz5@hotmail.com (B.D.D.C.); \\ gbotelho@quimica.uminho.pt (G.B.) \\ 4 Department of Chemistry, University of Trás-os-Montes e Alto Douro, 5000-801 Vila Real, Portugal \\ 5 BCMaterials, Basque Center for Materials, Applications and Nanostructures, UPV/EHU Science Park, \\ 48940 Leioa, Spain; senentxu.lanceros@bcmaterials.net \\ 6 Ikerbasque, Basque Foundation for Science, 48013 Bilbao, Spain \\ * Correspondence: d.correia@fisica.uminho.pt (D.M.C.); vbermude@utad.pt (V.d.Z.B.)
}

Received: 16 April 2020; Accepted: 18 May 2020; Published: 22 May 2020

\begin{abstract}
This work reports on the development of bending actuators based on poly(L-lactic acid) (PLLA)/ionic liquid (IL) blends, through the incorporation of $40 \% \mathrm{wt}$. of the 1-ethylmethylimidazolium bis(trifluoromethylsulfonyl)imide ([Emim][TFSI]) IL. The films, obtained by solvent casting at room temperature and $50{ }^{\circ} \mathrm{C}$, were subjected to several post-thermal treatments at $70,90,120$ and $140^{\circ} \mathrm{C}$, in order to modify the crystallinity of the films. The influence of the drying temperature and of [Emim][TFSI] blending on the morphological, structural, mechanical and electrical properties of the composite materials were studied. The IL induced the formation of a porous surface independently of the processing conditions. Moreover, the [Emim][TFSI] dopant and the post-thermal treatments at $70{ }^{\circ} \mathrm{C}$ promoted an increase of the degree of crystallinity of the samples. No significant changes were observed in the degree of crystallinity and Young Modulus for samples with thermal treatment between 70 and $140{ }^{\circ} \mathrm{C}$. The viability of the developed high ionic conductive blends for applications as soft actuators was evaluated. A maximum displacement of $1.7 \mathrm{~mm}$ was achieved with the PLLA/[Emim][TFSI] composite prepared at $50{ }^{\circ} \mathrm{C}$ and thermally treated $140{ }^{\circ} \mathrm{C}$, for an applied voltage of $10 \mathrm{Vpp}$, at a frequency of $100 \mathrm{mHz}$. This work highlights interesting avenues for the use of PLLA in the field of actuators.
\end{abstract}

Keywords: PLLA; ionic liquid; composites; degree of crystallinity; thermal treatments

\section{Introduction}

Poly(L-lactic acid) (PLLA) is a biodegradable and biocompatible thermoplastic polymer, which exhibits a wide variety of interesting features [1], including piezoelectricity [2]. On account of its wide versatility, PLLA has been extensively explored for the development of biomaterials targeting several biomedical applications, such as drug delivery [3,4] and tissue engineering [5-7]. Further, its potential for the fields of biosensors and actuators has been demonstrated [2,8,9].

Another very attractive feature offered by PLLA is the possibility of adjusting its degree of crystallinity by thermal annealing treatments and/or upon introduction of dopants, such as ionic liquids (ILs), which allow controlling its complex highly ordered structure, which is composed of intermingled crystalline and amorphous regions [2,9].

Typically, ILs are defined as salts composed solely by organic cations and organic or inorganic anions, with a melting point below $100{ }^{\circ} \mathrm{C}$. In recent years, due to their tunable properties 
and technological impact, such as high ionic conductivity [10], and high thermal, chemical and electrochemical stability [11-13], as well as low vapor pressure and volatility [14,15], ILs have been successfully employed in various domains for the design of numerous materials with enhanced features [16]. Additionally, because of their benign nature, ILs may play the role of green solvents with enormous potential in the field of sustainable chemistry $[17,18]$.

ILs have been incorporated into several host polymer matrices for the development of polymer/IL materials targeting a myriad of applications [16], including actuators [16,19-21]. The influence of different ILs, such as 1-butyl-3-methylimidazolium dibutylphosphate [22], 3-methyl-1-(ethoxycarbonylethyl)imidazolium tetrafluoroborate [23] and 1-butyl-3-methylimidazolium hexafluorophosphate [24] on the physical-chemical properties of PLLA were reported. However, to the best of our knowledge, the possibility of using PLLA/IL based materials in actuators has never been addressed so far.

In this work, PLLA and PLLA/IL films comprising the 1-ethyl-methylimidazolium bis(trifluoromethylsulfonyl)imide ([Emim][TFSI]) IL were developed by solvent casting to be applied as actuators. The films were prepared at different drying temperatures $\left(25\right.$ and $\left.50{ }^{\circ} \mathrm{C}\right)$, and subsequently subjected to four different post-thermal treatments in order to tune the degree of crystallinity, and, therefore, actuator performance. Their morphological, structural, mechanical, and electrical properties were characterized, and the influence of the post-thermal treatments investigated. Finally, the performance of the PLLA/IL composites as actuators was evaluated in terms of the influence of the drying temperature and post-thermal treatment temperatures on their bending response.

\section{Materials and Methods}

\subsection{Materials}

PLLA (Lumini L175, Corbion Purac, Montmeló, Spain; molecular weight (Mw): 217.000-225.000 $\mathrm{g} \mathrm{mol}^{-1}$, melting temperature: $175^{\circ} \mathrm{C}$, glass transition: $55-60{ }^{\circ} \mathrm{C}$, tensile modulus: $3500 \mathrm{MPa}$; tensile strength: $50 \mathrm{MPa}$ ), 1-ethyl-methylimidazolium bis(trifluoromethylsulfonyl)imide ([Emim][TFSI], 99\%, Iolitec, Heilbronn, Germany) and dichloromethane (DCM, 99\%, Merck, Darmstadt, Germany) were used as received.

\subsection{Preparation of the Neat and Composite PLLA-Based Films}

PLLA and PLLA/[Emim][TFSI] films were prepared using the solvent casting technique. To obtain the neat films, $1 \mathrm{~g}$ of PLLA was dissolved in $6.04 \mathrm{~mL}$ of DCM under magnetic stirring until complete dissolution. The solution was then cast onto a glass substrate and the solvent was evaporated (boiling temperature of $\mathrm{DCM} \approx 40^{\circ} \mathrm{C}$ ), either at room temperature (DT25, where DT stands for drying treatment) overnight, or at $50{ }^{\circ} \mathrm{C}$ (DT50) for $30 \mathrm{~min}$ (Figure 1). In the case of the preparation of the PLLA/[Emim][TFSI] films ( $40 \mathrm{wt} \%$ of IL), the IL was mixed with $6.04 \mathrm{~mL}$ of DCM. The [Emim][TFSI] was selected, attending to its good miscibility with the DMC and its electrical conductivity $(6.63 \mathrm{mS} / \mathrm{cm}$, data obtained from the provider). Moreover, a concentration of $40 \mathrm{wt} \%$ was used attending to the commonly maximum IL concentration used in IL/polymer blends for actuator applications, as reported in our previous studies [25]. Then, PLLA was added and after its complete dissolution, the resulting solution was spread onto a glass substrate and left to dry, either at room temperature or at $50{ }^{\circ} \mathrm{C}$ in an oven. The PLLA and PLLA/[Emim][TFSI] films were then subjected to different thermal treatments (TT) of 70, 90, 120 and $140{ }^{\circ} \mathrm{C}$ for $30 \mathrm{~min}$ [26]. These samples will be henceforth named TT 70, TT 90, TT 120 and TT 140, respectively. Neat PLLA and PLLA/[Emim][TFSI] films (DT25 and DT50) with a thickness of $\sim 40 \mu \mathrm{m}$ and $\sim 62 \mu \mathrm{m}$ were obtained (see Supplementary Information Table S1). 


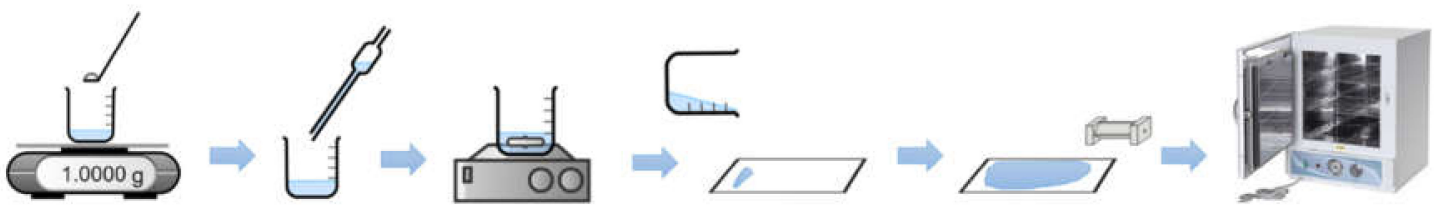

Figure 1. Schematic representation of the procedure used in the processing of the poly(L-lactic acid) (PLLA) and PLLA/[Emim][TFSI] films.

\subsection{Characterization}

\subsubsection{Morphological, Structural and Thermal Analyses}

The samples morphology was evaluated using a Scanning Electron Microscopy (SEM) in a NanoSEM-FEI Nova 200 (FEG/SEM) (FEI, Hillsboro, OR, USA), with an accelerating voltage of $10 \mathrm{kV}$. Prior to the analysis the samples were coated with gold ( $\mathrm{Au}$ ) by magnetron sputtering (Polaron SC502). The attenuated total reflection (ATR)/Fourier transform infrared spectroscopy (FTIR) spectra were recorded with a Jasco FT/IR-4100 FTIR (Jasco, Pfungstadt, Germany) spectrometer, at room temperature in the 4000 and $600 \mathrm{~cm}^{-1}$ range, using 64 scans and a resolution of $4 \mathrm{~cm}^{-1}$. DSC measurements were performed using a DSC 200 F3 Maia Netzsch calorimeter from NETZSCH Premier Technologies (Iphofen, Germany), between 30 and $220^{\circ} \mathrm{C}$ at a heating rate of $10^{\circ} \mathrm{C} \mathrm{min}-1$ in a nitrogen atmosphere. For the measurements, approximately $4 \mathrm{mg}$ of each sample was used. The degree of crystallinity $\left(X_{c}\right)$ was calculated using Equation (1):

$$
X_{c}(\%)=\frac{\Delta H}{\Delta H^{o} m} \times 100
$$

where $\Delta \mathrm{H}$ is the melting enthalpy, and $\Delta H^{o} m$ is the melting enthalpy, for a fully crystallized PLLA sample (93.1 $\mathrm{J} \mathrm{g}^{-1}$ [26]). The mechanical measurements were carried out in the tensile mode at room temperature in triplicate for each processing condition, in a universal testing machine (model AG-IS), from Shimadzu (Kyoto, Japan), using a load cell of $50 \mathrm{~N}$. The samples, with a rectangular shape $(30 \mathrm{~mm} \times 10 \mathrm{~mm})$, were analyzed at a speed of $1 \mathrm{~mm} \mathrm{~min}^{-1}$.

\subsubsection{Electrical and Electromechanical Characterization}

The electrical measurements were performed with a Keithley 6430 (Allied, Las Vegas, NV, USA) picoammeter/voltage source. Prior to the experiments, the samples circular Au electrodes of $5 \mathrm{~mm}$ diameter were deposited in parallel in both sides of the samples by magnetron sputtering (Polaron Coater SC502, Quorum, Lewes, UK), thus ensuring a good electric contact between the sample and sample holder. The volume d.c. electrical conductivity was obtained at room temperature from the characteristic I-V curves, where the current $(\mathrm{I})$ and voltage $(\mathrm{V})$ were measured between -3 and $+3 \mathrm{~V}$ and the conductivity $(\sigma)$ was calculated considering the geometrical characteristics of the samples according to Equation (2):

$$
\sigma=\frac{1}{\left(\frac{R A}{L}\right)}
$$

where $R$ is the electrical resistance, $L$ is the sample thickness and $A$ is the area of the electrodes. The performance of the films as actuators was evaluated by bending tests in a home-made samples holder [25]. Prior to the measurements, the samples with dimensions of $12 \mathrm{~mm} \times 2 \mathrm{~mm}$ were covered with Au on both sides by magnetron sputtering (Polaron SC502). The needles of the sample holder, clamping the bottom of the samples, were connected to an Agilent 33220A (Allied, Las Vegas, NV, USA) function generator. The displacement $(\delta)$ of the sample tip was measured by applying a square wave signal with peak-to-peak voltages (Vpp) of 5 and $10 \mathrm{~V}$, at a frequency of $100 \mathrm{mHz}$. 


\section{Results and Discussion}

\subsection{Morphology}

The morphology of the PLLA and PLLA/[Emim][TFSI] films obtained at a room temperature and at $50{ }^{\circ} \mathrm{C}$ was evaluated by SEM. The cross-section images of the films are presented in Figure 2.
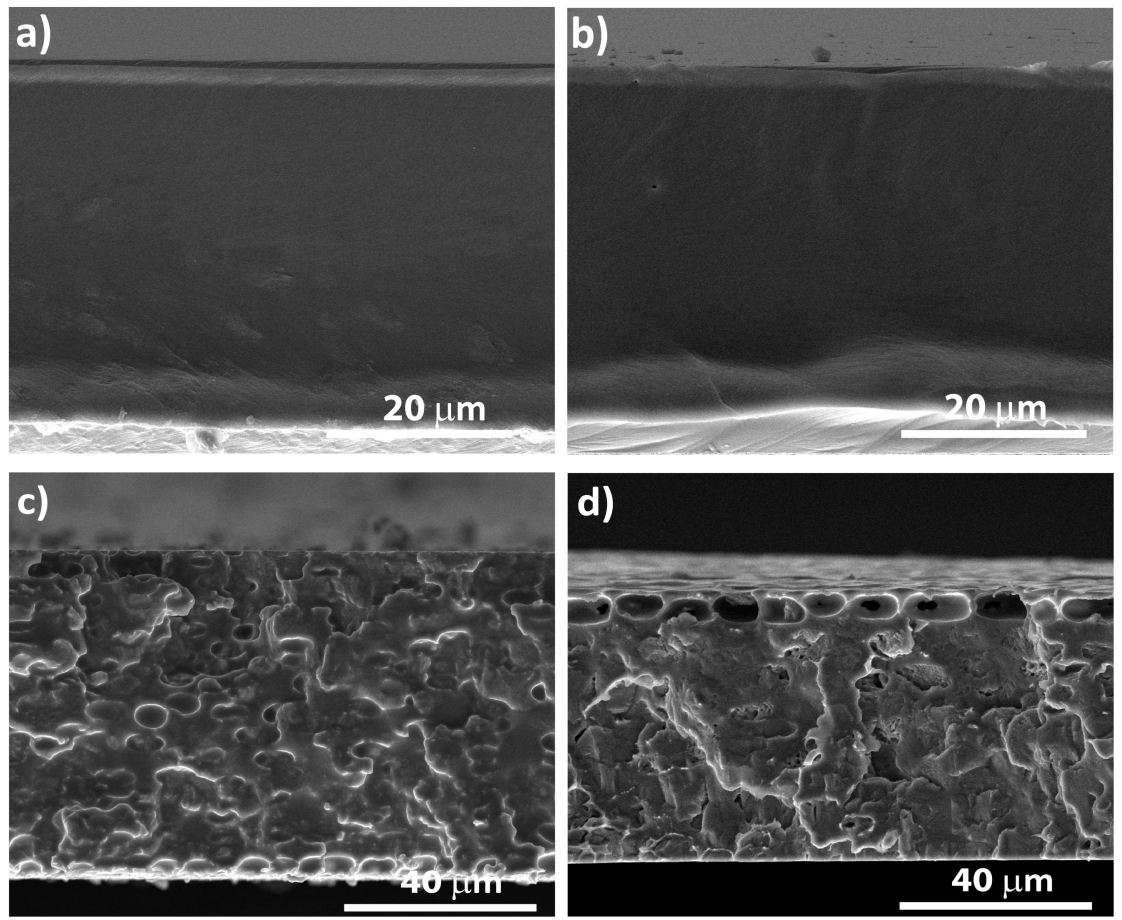

Figure 2. Cross-section SEM images of the PLLA-based films: (a) PLLA-DT25, (b) PLLA-DT50, (c) PLLA/[Emim][TFSI]-DT25 and (d) PLLA/[Emim][TFSI]-DT50.

Independently of the solvent evaporation temperature, the PLLA films exhibited a homogenous and non-porous texture, as shown in the cross-section SEM images (Figure 2a,b). Figure 2c,d demonstrate that upon [Emim][TFSI] incorporation into the PLLA matrix, and independently of the drying temperature, the IL induced the occurrence of a significant roughness and porosity. This effect was reported previously for other polymeric matrices (e.g., poly(vinylidene fluoride) (PVDF) [27]), and was attributed to the strong [Emim][TFSI]-DCM interaction, phase separation within the polymer solutions and [Emim][TFSI] trapping within the pores, once the solvent is evaporated from the solvent+IL rich regions [28].

\subsection{Physical-Chemical Characterization}

To evaluate possible chemical modifications occurring in the PLLA polymer structure depending on the film processing at different drying temperatures, and to determine the effect of blending PLLA with [Emim][TFSI], all samples were analyzed by ATR/FTIR spectroscopy. The ATR/FTIR spectra are reproduced in Figure 3. The assignment of the main vibration bands is given in Table 1.

The ATR/FTIR spectra of the PPLA-DT25 and PLLA-DT50 films display the main characteristic absorption bands of PLLA. No relevant differences are observed, depending on the processing temperature. The bands observed at 865 and $754 \mathrm{~cm}^{-1}$ are attributed to the stretching vibrations of the $\mathrm{C}-\mathrm{C}(=\mathrm{O}) \mathrm{O}$ and $\mathrm{C}=\mathrm{O}$ moieties of the ester group, respectively [29,30]. The absorption band detected at $955 \mathrm{~cm}^{-1}$ is assigned to the $\mathrm{C}-\mathrm{C}$ and $\mathrm{CH}_{3}$ stretching vibrations. The absorption band at $1080 \mathrm{~cm}^{-1}$ corresponds to the asymmetric stretching of the $\mathrm{C}-\mathrm{O}-\mathrm{C}$ vibration. Finally, the most relevant bands between 2999 and $1750 \mathrm{~cm}^{-1}$ corresponds to the stretching vibrations of the $\mathrm{CH}_{3}$ and $\mathrm{C}=\mathrm{O}$ groups, respectively $[30,31]$. 


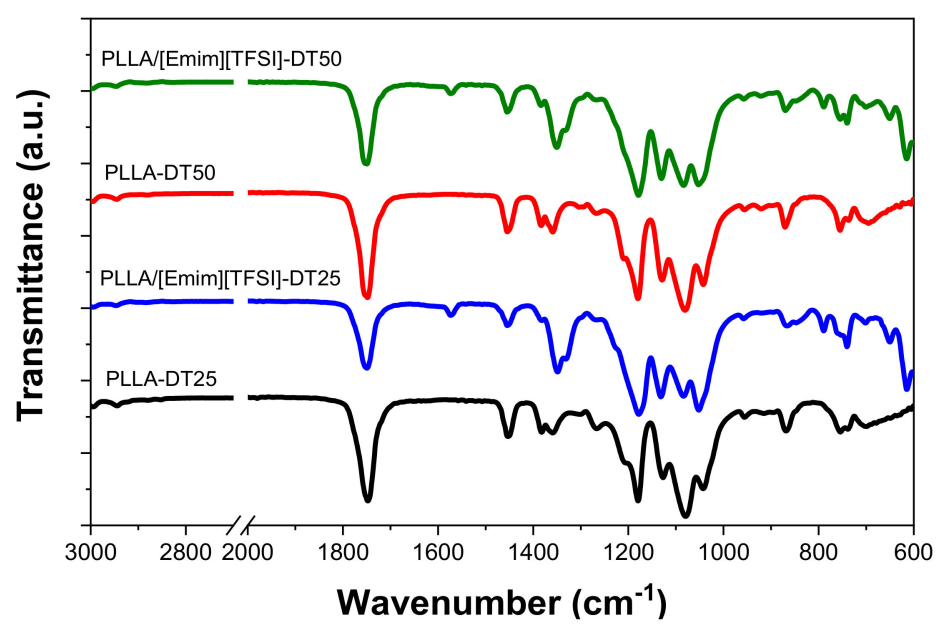

Figure 3. ATR/FTIR spectra of the PLLA and PLLA/[Emim][TFSI] films obtained at different drying temperatures.

Table 1. Main absorption bands of PLLA and [Emim][TFSI] in the prepared samples.

\begin{tabular}{|c|c|c|}
\hline \multirow{2}{*}{$\begin{array}{l}\text { Wavenumbers } \\
\quad\left(\mathrm{cm}^{-1}\right)\end{array}$} & \multicolumn{2}{|c|}{ Attribution } \\
\hline & PLLA [29] & [Emim][TFSI] [32] \\
\hline 2997 & $\mathrm{CH}_{3}$ asymmetric stretching & - \\
\hline 1752 & $\mathrm{C}=\mathrm{O}$ stretching & - \\
\hline 1570 & - & $\begin{array}{l}\text { ring vibration } \mathrm{N}-\mathrm{CH}_{3} \text { and } \\
\mathrm{N}-\mathrm{CH}_{2}-\mathrm{CH}_{3}\end{array}$ \\
\hline 1454 & $\mathrm{CH}_{3}$ asymmetric stretching & - \\
\hline 1383 & $\mathrm{CH}_{3}$ symmetric stretching & - \\
\hline 1358 & $\mathrm{CH}$ stretching $+\mathrm{CH}_{3}$ symmetric stretching & - \\
\hline 1348 & & $\mathrm{SO}_{2}$ symmetric stretching \\
\hline 1300 & $\mathrm{CH}$ stretching & - \\
\hline 1184 & $\mathrm{C}-\mathrm{O}-\mathrm{C}+\mathrm{CH}_{3}$ asymmetric stretching & $\mathrm{CF}_{3}$ antisymmetric stretching \\
\hline 1132 & $\mathrm{CH}_{3}$ asymmetric stretching & $\mathrm{SO}_{2}$ symmetric stretching \\
\hline 1085 & C-O-C symmetric stretching & - \\
\hline 1045 & $\mathrm{C}-\mathrm{CH}_{3}$ stretching & \\
\hline 1051 & & $\mathrm{C}-\mathrm{C}$ and $\mathrm{NCH}_{3}$ stretching \\
\hline 921 & $\mathrm{C}-\mathrm{C}$ stretching $+\mathrm{CH}_{3}$ rotation & - \\
\hline 871 & $\mathrm{C}-\mathrm{COO}$ stretching & \\
\hline 738 & - & $\mathrm{CF}_{3}$ stretching \\
\hline 755 & $\mathrm{C}=\mathrm{O}$ stretching & - \\
\hline 740 & & $\mathrm{CF}_{3}$ symmetric stretching \\
\hline 651 & & S-N-S bending \\
\hline 619 & - & $\mathrm{SO}_{2}$ antisymmetric bending \\
\hline
\end{tabular}

The main absorption bands of pristine PLLA are also found in the ATR/FT-IR spectra of the PLLA/[Emim][TFSI] blends. These spectra also include the characteristic absorption bands of the IL at 1570 and $610 \mathrm{~cm}^{-1}$ assigned to the vibration of the $\mathrm{N}-\mathrm{CH}_{3}$ and $\mathrm{N}-\mathrm{CH}_{2}-\mathrm{CH}_{3}$ groups of the imidazolium ring, and to the $\mathrm{CF}_{3}$ groups of the TFSI ${ }^{-}$anions, respectively. The intensity increase of the band at $1350 \mathrm{~cm}^{-1}$ is indicative of the overlapping of the absorption bands from both components of the blend [32]. 
The influence of the [Emim][TFSI] incorporation and the effect of the drying treatment on the thermal properties and degree of crystallinity of PLLA and [Emim][TFSI] films was evaluated by DSC. Figure 4 shows the DSC curves obtained for all the samples before and after the drying treatment.
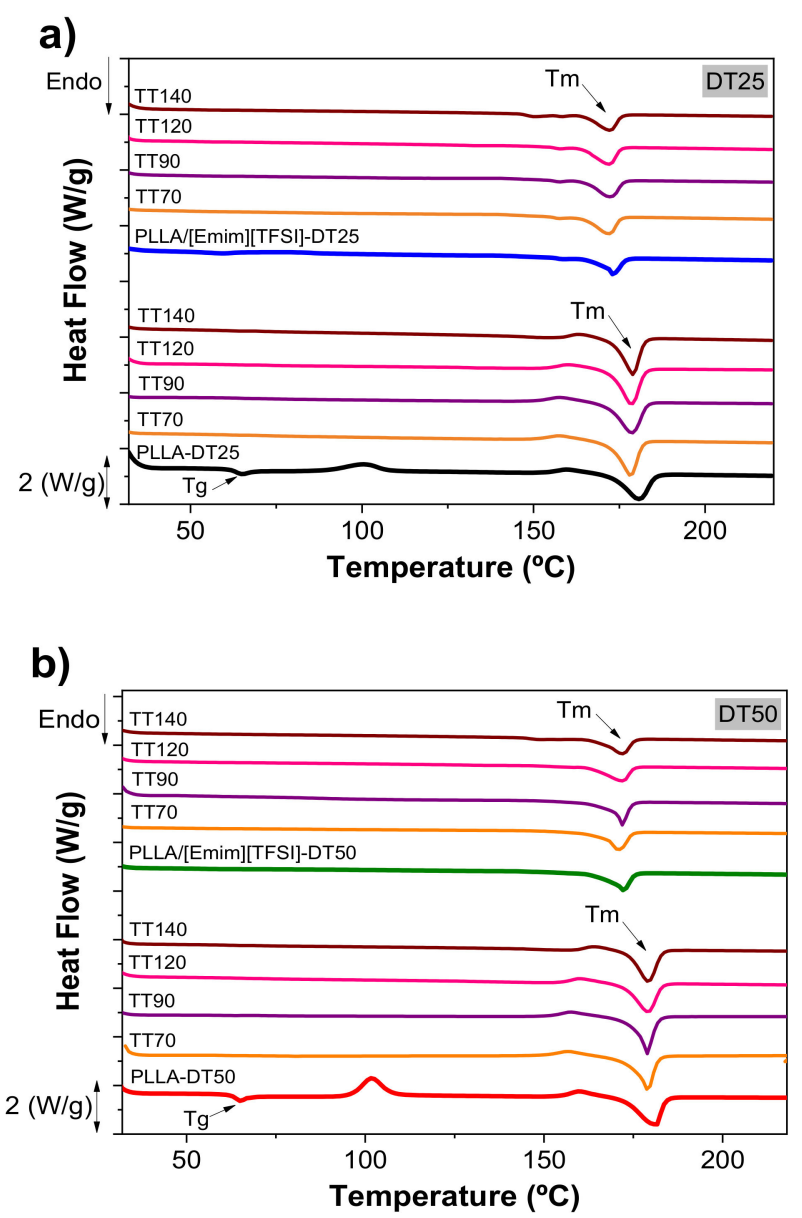

Figure 4. DSC curves as a function of the post-thermal treatment temperature for the PLLA-based films dried at: (a) $25^{\circ} \mathrm{C}$ and (b) $50{ }^{\circ} \mathrm{C}$.

Both pristine PLLA samples, i.e., PPLA-DT25 and PPLA-DT50, produced endothermic peaks at $\sim 67$ and $\sim 172{ }^{\circ} \mathrm{C}$, respectively (Figure $4 \mathrm{a}, \mathrm{b}$ ), associated with the PLLA glass transition $\left(\mathrm{T}_{\mathrm{g}}\right.$ ) and melting $\left(\mathrm{T}_{\mathrm{m}}\right)$ temperatures, respectively [30,31]. The exothermic peak appearing between 100 and $160{ }^{\circ} \mathrm{C}$ is related to the polymer cold crystallization [30,31].

Upon addition of [Emim][TFSI] to the PLLA matrix, and independently of the drying temperature, two effects are evident. Figure $4 \mathrm{a}, \mathrm{b}$ demonstrate that the events attributed to the glass transition and cold crystallization disappeared. In contrast, no significant changes were observed in the $\mathrm{T}_{\mathrm{m}}$ value of the polymer.

The influence of the post-thermal treatment performed on the degree of crystallinity was further assessed. Figure 4 allows inferring that a slight decrease in the $T_{m}$ value of PLLA was observed for the post-thermal treatment carried out at $70{ }^{\circ} \mathrm{C}$. The use of higher post-thermal treatment temperatures did not cause, however, any significant changes in $\mathrm{T}_{\mathrm{m}}$.

The effect of [Emim][TFSI] blending and post-thermal treatments on the degree of crystallinity were quantitatively evaluated by means of Equation (1), on the basis of the DSC data. The results are shown in Figure 5 and collected in Table S1. 

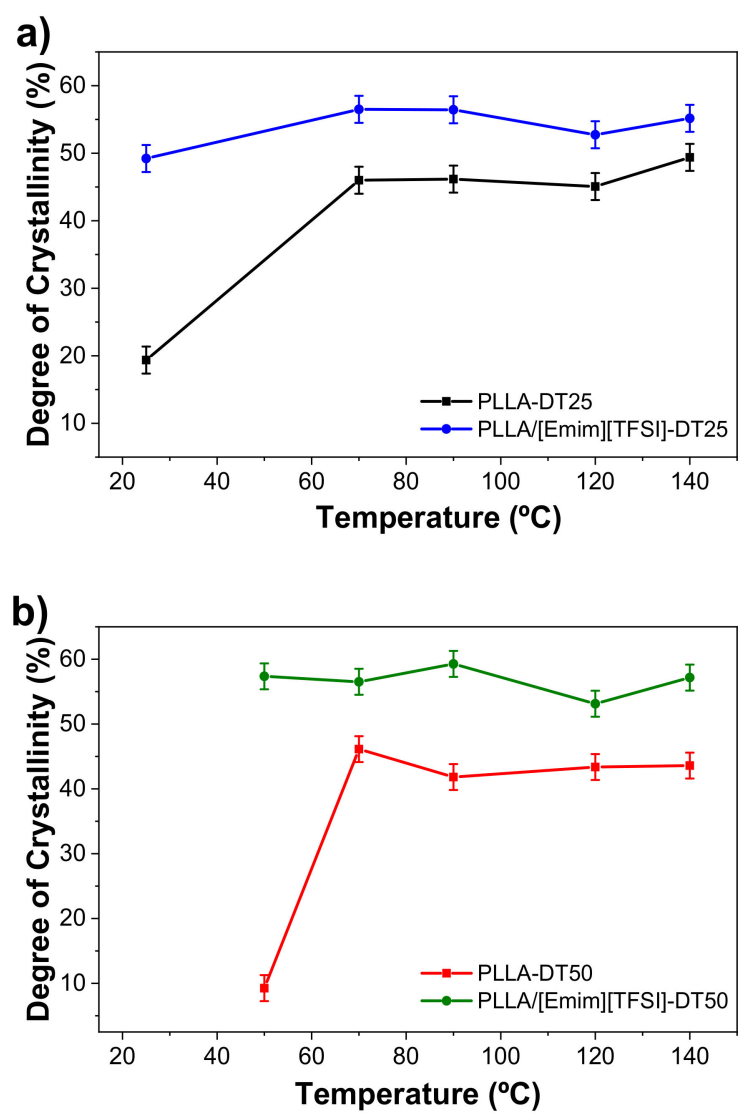

Figure 5. Degree of crystallinity as a function of the post-thermal treatment temperature for the PLLA-based films dried at: (a) $25^{\circ} \mathrm{C}$; and (b) $50{ }^{\circ} \mathrm{C}$.

Table S1 allows inferring that a significant increase of the degree of crystallinity from ca. 19 to $49 \%$ resulted upon IL incorporation for the samples dried at $25^{\circ} \mathrm{C}$. Similarly, an increase of crystallinity was also observed for the samples dried at $50{ }^{\circ} \mathrm{C}$ (from ca. 9 to $57 \%$ ). The similar trend observed for both composites at different drying temperatures indicates that the IL acts as a nucleating agent, inducing the formation of a higher number of nucleation centers accelerating the crystallization process [33]. This influence of the IL in the crystallization process of a polymer matrix has been reported for related polymer/IL blends [34].

Likewise, the post-thermal treatments promoted an important increase in the degree of crystallinity of the samples (Table S1). In all cases, this increase was more marked for the post-thermal treatment performed at $70{ }^{\circ} \mathrm{C}$ : from $19 \%$ to $46 \%$ and from $49 \%$ to $56 \%$ in the case of PLLA-DT25 and PLLA/[Emim][TFSI]-DT25, respectively. For the samples dried at $50{ }^{\circ} \mathrm{C}$ (PLLA-DT50 and PLLA/[Emim][TFSI]-DT50), an increase also resulted, but the maximum values of the degree of crystallinity remained practically the same as those of the samples dried at room temperature. In contrast, the increase of the post-thermal treatment temperature from 70 to $140{ }^{\circ} \mathrm{C}$ does not promote relevant variations in the degree of crystallinity, obtaining values of $46 \%$ and $44 \%$, respectively.

The highest degree of crystallinity values is observed in the polymer blends, as a result of the higher number of nucleation centers induced by the presence of the IL. Upon heating, the growth of nucleation centers led to a higher degree of crystallinity [26].

\subsection{Mechanical Properties}

The mechanical properties of the samples were determined by uniaxial stress-strain measurements. The goal was to evaluate the effect of the drying treatment, [Emim][TFSI] addition, and post-thermal treatments on the Young modulus $(E)$. The results of the tensile stress measurements for PLLA and the 
PLLA/[Emim][TFSI] films dried at 25 and $50{ }^{\circ} \mathrm{C}$, before and after the post-thermal treatments at 70 and $140^{\circ} \mathrm{C}$, are reproduced in Figure 6. The $E$ values of all the samples are given in Table S2.
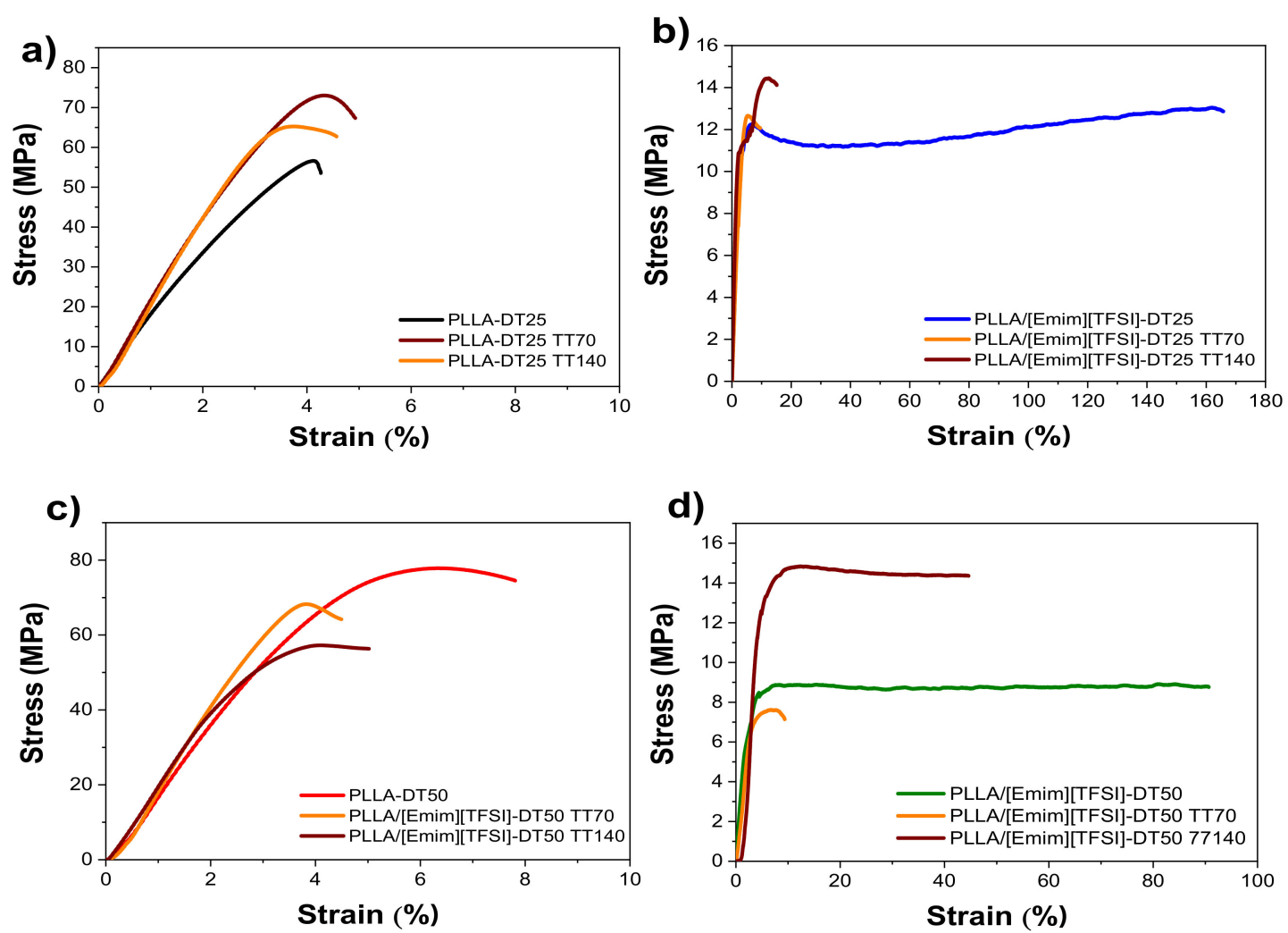

Figure 6. Stress vs. strain curves of PLLA and PLLA/[Emim][TFSI] films dried at $25^{\circ} \mathrm{C}(\mathbf{a}$ and $\mathbf{b})$ and $50{ }^{\circ} \mathrm{C}$ (c and d), for samples subjected to post-thermal treatment at 70 and $140{ }^{\circ} \mathrm{C}$.

Figure 6 demonstrates that, independently of the drying temperature, the PLLA films exhibited behavior typical of a thermoplastic polymer [35]. The [Emim][TFSI] added to the PLLA matrix exerted a plasticizing effect manifested in a noticeable elastic deformation region, followed by yielding and a linear regime. Additionally, it is also observed that, independently of the drying temperature, the IL incorporation into the PLLA matrix induces an increase in the elongation at break and ductility. The nominal $E$ value was deduced from the linear regime of the elastic region and using the tangent method (Figure 6 and Table S2).

Figure 6 and Table S2 allow one to conclude that the drying temperature slightly influenced the $E$ value of the samples, increasing from $1410 \pm 340 \mathrm{MPa}$ (PLLA-DT25) to $1820 \pm 280 \mathrm{MPa}$ (PLLA-DT50). For the PLLA/[Emim][TFSI] films, a marked decrease of the $E$ value occurred in PLLA/[Emim][TFSI]-DT25 $(390 \pm 160 \mathrm{MPa})$ and PLLA/[Emim][TFSI]-DT50 (380 $\pm 80 \mathrm{MPa})$. This decrease may be correlated with the plasticizing effect of [Emim][TFSI] $[20,25]$. For the PLLA samples dried at room temperature, no relevant changes are observed in $E$ value when the post-thermal treatment temperature was increased from $70{ }^{\circ} \mathrm{C}(1840 \pm 330 \mathrm{MPa})$ to $140{ }^{\circ} \mathrm{C}(2000 \pm 100 \mathrm{MPa})$. However, for samples post-treated at 90 and $120^{\circ} \mathrm{C}$, a slight decrease of $E$ is observed, being more noticeable for the samples post-thermal treated at $120^{\circ} \mathrm{C}$. This fact is associated to the slight decrease of the degree of crystallinity, as shown in Table S2, decreasing the stiffness of the samples.

In the case of the PLLA/[Emim][TFSI]-DT25 sample, a small increase from $390 \pm 160 \mathrm{MPa}$ (without post-treatment) to $550 \pm 90 \mathrm{MPa}$ (PLLA/[Emim][TFSI]-DT25-TT70) was followed by a decrease for PLLA/[Emim][TFSI]-DT25-TT90 and PLLA/[Emim][TFSI]-DT25-TT120. The PLLA/[Emim][TFSI] sample treated at $140{ }^{\circ} \mathrm{C}$ exhibited an $E$ value of $560 \pm 90 \mathrm{MPa}$. A similar trend was observed for 
the PLLA-DT50 and PLLA/[Emim][TFSI]-DT50 materials. The Young Modulus behavior for all the samples and the effect of thermal treatments are summarized in Figure 7 and Table S3.

a)

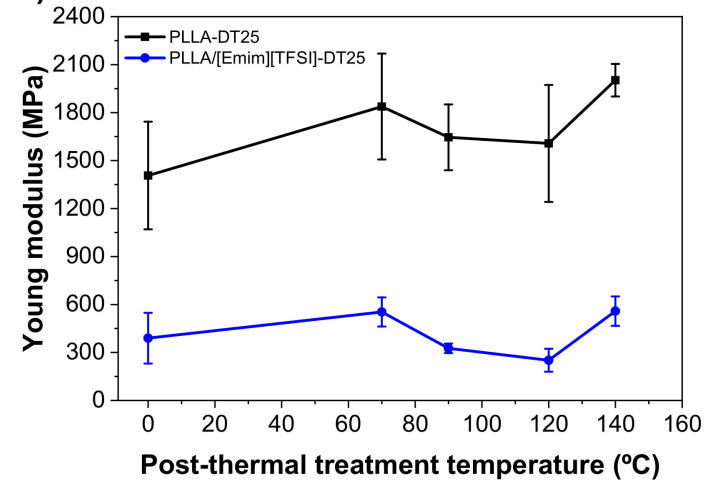

b)

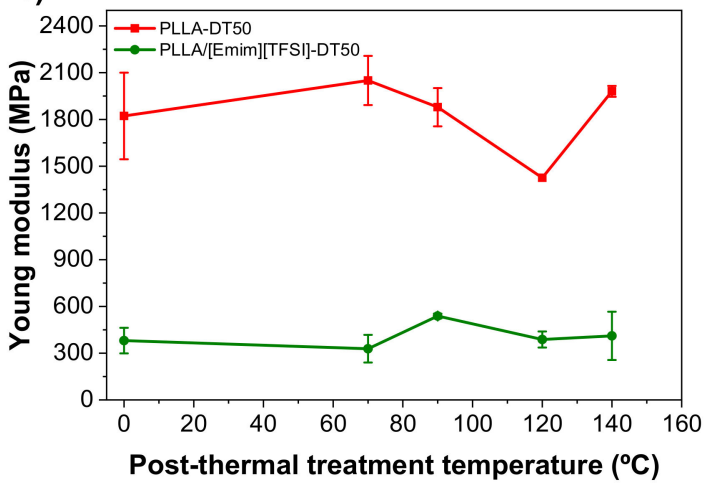

Figure 7. Young modulus as a function of the post-thermal treatment temperature for PLLA and PLLA/[Emim][TFSI] films dried at: (a) $25^{\circ} \mathrm{C}$ and (b) $50^{\circ} \mathrm{C}$.

\subsection{Electrical Properties}

The influence of [Emim][TFSI] on the electrical properties of the PLLA/[Emim][TFSI] composites was evaluated. Additionally, the influence of the post-thermal treatments was also investigated for the samples post-treated with the minimum and maximum temperatures $\left(70\right.$ and $\left.140{ }^{\circ} \mathrm{C}\right)$.

Figure $8 \mathrm{a}, \mathrm{b}$ show that the samples dried at 25 and $50{ }^{\circ} \mathrm{C}$ evidenced, as expected, a linear relation between I and V. The electrical conductivity of the samples was evaluated using Equation (2) and the results are reproduced in Figure 8c,d.
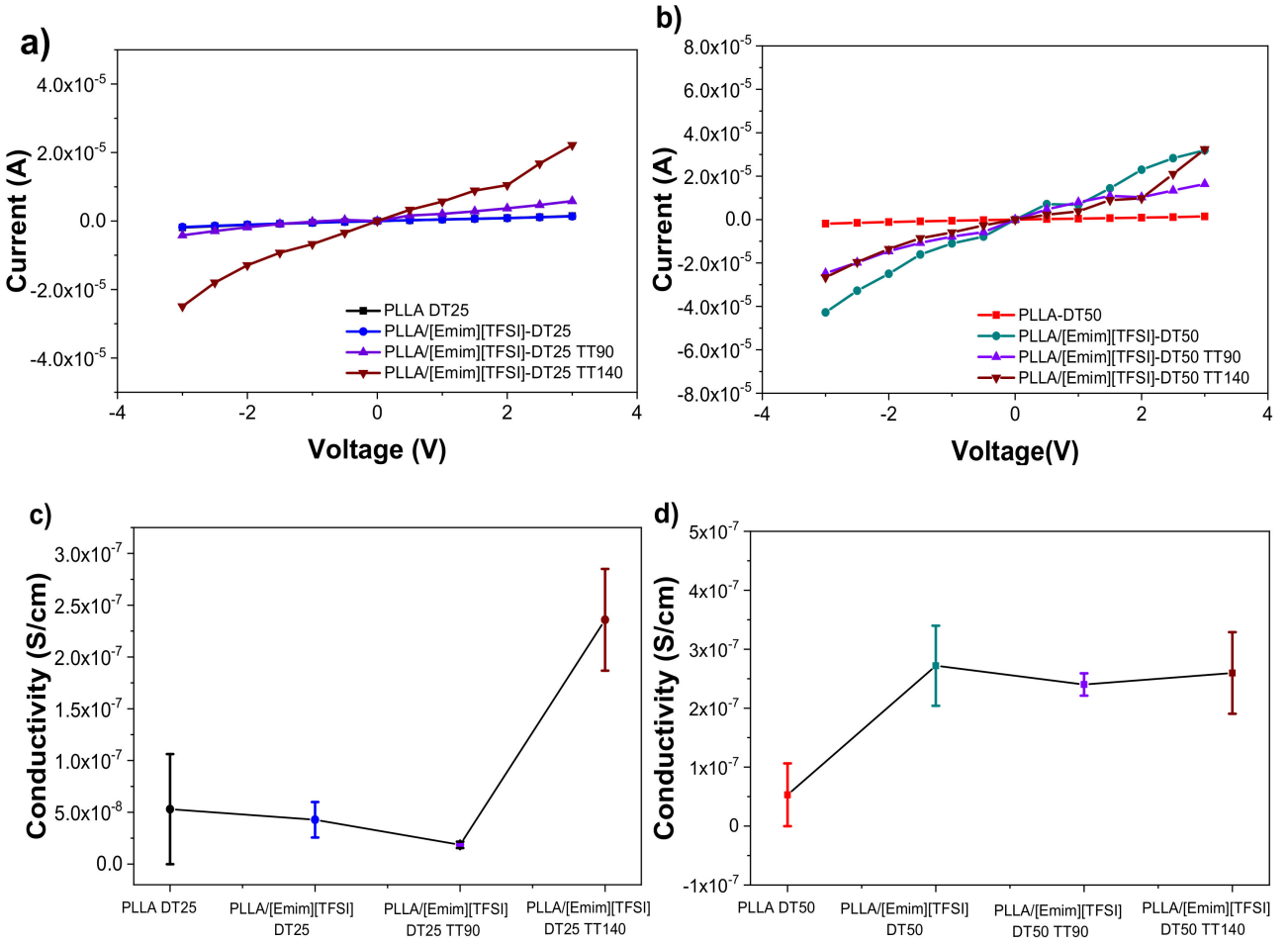

Figure 8. IV curves $(\mathbf{a}, \mathbf{b})$ and electrical conductivity $(\mathbf{c}, \mathbf{d})$ as a function of the post-thermal treatment temperature of the PLLA-based films dried at $25^{\circ} \mathrm{C}$ (left column) and $50{ }^{\circ} \mathrm{C}$ (right column).

In the case of the samples dried at room temperature, the conductivity remained practically the same $\left(5.30 \times 10^{-8}\right.$ to $4.28 \times 10^{-8} \mathrm{~S} \mathrm{~cm}^{-1}$ for PPLA-DT25 and PLLA/[Emim][TFSI]-DT25, 
respectively). The post-thermal treatment at $90^{\circ} \mathrm{C}$ led to a slight decrease to $1.86 \times 10^{-8} \mathrm{~S} \mathrm{~cm}^{-1}$ for the PLLA/[Emim][TFSI]-DT25. However, the electrical conductivity of [Emim][TFSI]/PLLA-DT25TT140 increased substantially to $2.36 \times 10^{-5} \mathrm{~S} / \mathrm{m}$, probably because at this temperature (close to the $\mathrm{T}_{\mathrm{m}}$ of PLLA), the energy supplied induced the optimal orientation of the polymeric chains, promoting ion transport.

In the case of the samples dried at $50{ }^{\circ} \mathrm{C}$ (Figure 8d), a significant increase in the electrical conductivity from $5.30 \times 10^{-8}$ to $2.72 \times 10^{-7} \mathrm{~S} \mathrm{~cm}^{-1}$ resulted from the addition of the [Emim][TFSI] to the host matrix [20]. The post-thermal treatments performed at 90 and $140{ }^{\circ} \mathrm{C}$ exerted no significant changes in the electrical conductivity. Furthermore, one should notice that no significant changes occur in the electrical conductivity of [Emim][TFSI]/PLLA-DT50 before and after the post-thermal treatments. These results are associated to the similar degree of crystallinity of the samples for a given number of charge carriers, in which the amorphous phase plays a determinant role into the ionic carriers transport $[20,36]$.

\subsection{Electromechanical Measurements}

The potential of the developed composites for the development of biocompatible soft actuators was evaluated by electromechanical measurements. Considering the high electrical conductivity measured for PLLA/[Emim][TFSI]-DT25 and PLLA/[Emim][TFSI]-DT50 post-treated at $140{ }^{\circ} \mathrm{C}$, the electromechanical measurements were focused exclusively on these samples. The displacement as a function of time upon an applied voltage of 5 and $10 \mathrm{Vpp}$ at a frequency of $100 \mathrm{mHz}$ is represented in Figure 9.

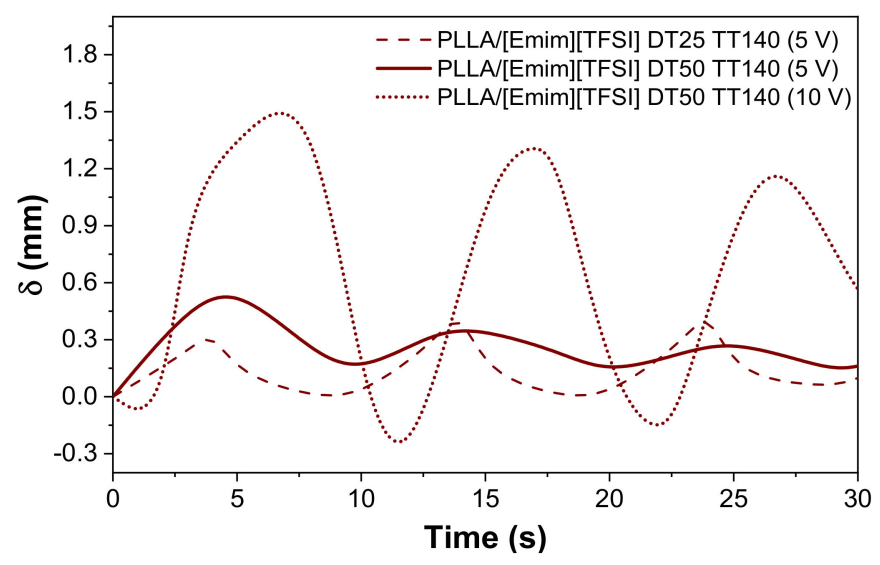

Figure 9. Displacement as a function of time for the PLLA/[Emim][TFSI]-DT25 and PLLA/[Emim][TFSI]-DT50 samples, subjected to a post-thermal treatment at $140{ }^{\circ} \mathrm{C}$.

The observed displacements $(\delta)$ were measured from the position of the actuator tip upon an applied voltage of $5 \mathrm{Vpp}$ at a frequency of $100 \mathrm{mHz}$. Figure 9 shows that for an applied voltage of $5 \mathrm{Vpp}$, the PLLA/[Emim][TFSI]-DT25 film exhibited a maximum displacement ranging from $\sim 0.3$ to $0.4 \mathrm{~mm}$. The maximum displacement was observed for the PLLA/[Emim][TFSI]-DT50 composite, with values ranging between 0.2 and $0.5 \mathrm{~mm}$. For these samples, with the increase in the applied voltage from 5 to $10 \mathrm{Vpp}$, an increase in the displacement to $1.7 \mathrm{~mm}$ resulted (Figure 10a), indicating that high voltages favored the ions' movement within the polymer matrix [19]. The strain developed as a response to the applied electrical field results from the diffusion of the ions and migration to the positive (anions) and negative (cations) electrode layers, and subsequent accumulation close to the electrodes [19,37] (Figure 10b).

It must be also noticed that the displacement does not follow a symmetric behavior, which is manifested by the non-symmetric displacement curves with respect to the initial position, as a result of the irreversible movement of ions and relaxation. Upon voltage application, due to the different 
cation and anion sizes, an imbalance of the ion transport occurs, leading to a non-symmetrical ions dynamical behavior (Figure 10b) [19].

a)
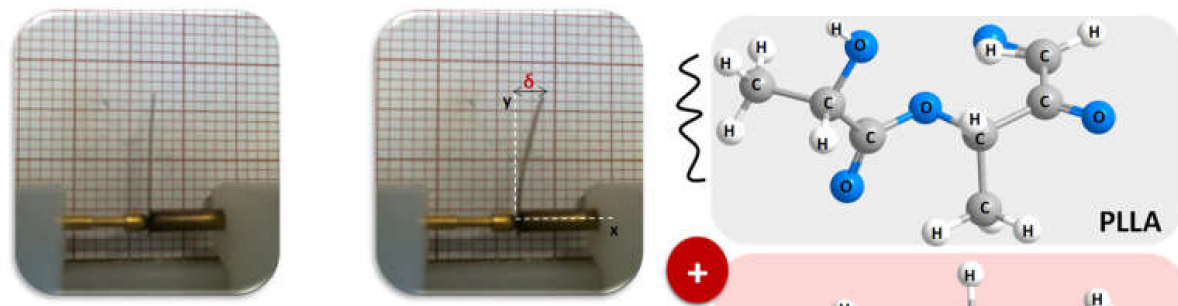

b)
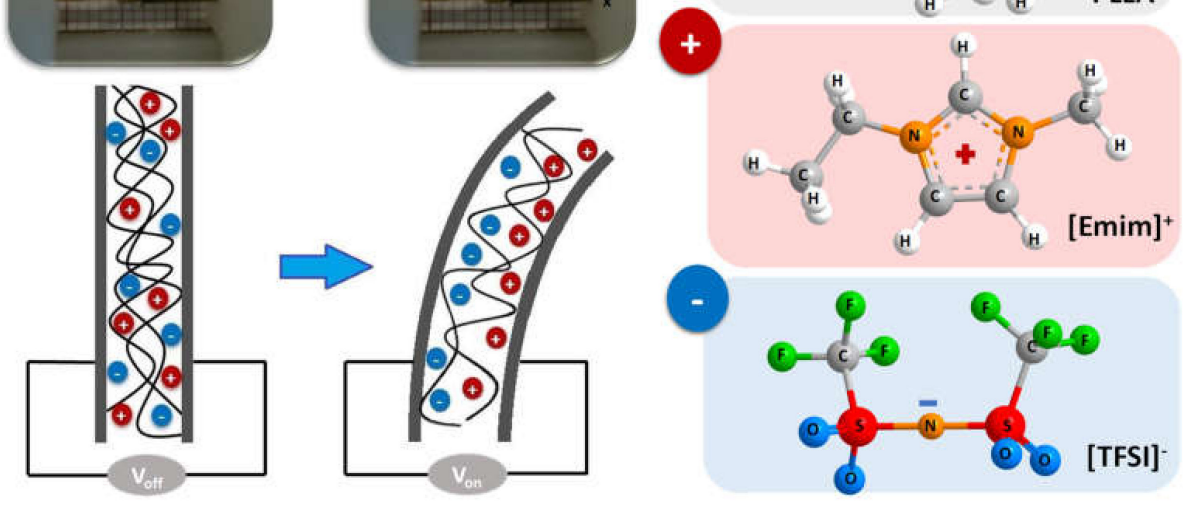

Figure 10. (a) Displacement observed for the PLLA/[Emim][TFSI]-DT25 TT140, upon an applied voltage of $10 \mathrm{Vpp}$ at a frequency of $100 \mathrm{mHz}$; (b) Schematic representation of the ionic movement caused by an applied voltage.

\section{Conclusions}

The PLLA polymer and PLLA/[Emim][TFSI] composites were obtained by solvent casting at different drying temperatures $\left(25\right.$ and $\left.50{ }^{\circ} \mathrm{C}\right)$. Additionally, the films were subjected to different post-thermal treatments, to study their influence on the degree of crystallization of pristine and composite films. The effect of the drying temperature on the morphological and chemical properties was evaluated. Independently of the drying temperature, the incorporation of [Emim][TFSI] into the PLLA matrix promoted the formation of a porous texture. The degree off crystallinity increased with [Emim][TFSI] addition, and with post-thermal treatments performed at $70{ }^{\circ} \mathrm{C}$. No significant changes occurred with the increase of the post-thermal treatment temperature from 70 to $140{ }^{\circ} \mathrm{C}$. With respect to mechanical measurements, [Emim][TFSI] acted as a plasticizer, decreasing the Young Modulus. No significative changes are observed in the Young modulus for the samples dried at room temperature and post-thermal treated at temperatures ranging from $70{ }^{\circ} \mathrm{C}$ to $140{ }^{\circ} \mathrm{C}$. Electrical conductivity measurements revealed an increase in the conductivity values with [Emim][TFSI] blending and post-thermal treatments. We demonstrate here that the developed high ionic conductivity blends offer great potential as soft actuators. A maximum displacement of $1.7 \mathrm{~mm}$ was measured for the sample PLLA/[Emim][TFSI]-DT50-TT140 for an applied voltage of $10 \mathrm{Vpp}$, at a frequency of $100 \mathrm{mHz}$.

Supplementary Materials: The following are available online at http://www.mdpi.com/2073-4360/12/5/1187/s1, Table S1, Thickness of the PLLA and PLLA/IL films as a function of the drying and post-treatment temperature, Table S2: Degree of crystallinity of the PLLA and PLLA/[Emim][TFSI] films as a function of drying temperature and post-thermal treatment; Table S3: Young modulus for PLLA and PLLA/[Emim][TFSI] films.

Author Contributions: Conceptualization, S.L.-M. and D.M.C.; methodology, S.L.-M., G.B. and D.M.C.; validation, S.L.-M.; formal analysis, D.M.C., S.L.-M., G.B. and V.d.Z.B.; investigation, B.D.D.C., D.M.C. and L.C.F.; resources, S.L.-M., G.B. and V.d.Z.B.; data curation, D.M.C., B.D.D.C. and L.C.F.; writing-original draft preparation, D.M.C. and L.C.F.; writing-review and editing, S.L.-M., G.B. and V.d.Z.B.; funding acquisition, S.L.-M. and G.B. All authors have read and agreed to the published version of the manuscript.

Funding: The authors thank the FCT—Fundação para a Ciência e Tecnologia-for financial support under the Strategic Funding UID/FIS/04650/2020, and PEST-C/QUI/UIO686/2019, the Associated Laboratory Research Unit for Green Chemistry, Technologies and Clean Processes, LAQV (financed by national funds from FCT/MEC, UID/QUI/50006/2020 and ERDF under the PT2020, POCI-01-0145-FEDER-007265) and projects 
PTDC/BTM-MAT/28237/2017 and PTDC/EMD-EMD/28159/2017. DMC and LCF also thank the grants SFRH/BPD/121526/2016 and SFRH/BD/145345/2019, respectively.

Acknowledgments: The authors acknowledge funding from the Basque Government Industry and Education Departments under the ELKARTEK, HAZITEK and PIBA (PIBA-2018-06) programs, respectively. Technical and human support provided by SGIker (UPV/EHU, MICINN, GV/EJ, EGEF and ESF) is gratefully acknowledged.

Conflicts of Interest: The authors declare no conflict of interest.

\section{References}

1. Farah, S.; Anderson, D.G.; Langer, R. Physical and mechanical properties of PLA, and their functions in widespread applications-A comprehensive review. Adv. Drug Deliv. Rev. 2016, 107, 367-392. [CrossRef]

2. Chorsi, M.T.; Curry, E.J.; Chorsi, H.T.; Das, R.; Baroody, J.; Purohit, P.K.; Ilies, H.; Nguyen, T.D. Piezoelectric Biomaterials for Sensors and Actuators. Adv. Mater. 2019, 31, 1802084. [CrossRef]

3. Tyler, B.; Gullotti, D.; Mangraviti, A.; Utsuki, T.; Brem, H. Polylactic acid (PLA) controlled delivery carriers for biomedical applications. Adv. Drug Deliv. Rev. 2016, 107, 163-175. [CrossRef] [PubMed]

4. Hyon, S.H. Biodegradable poly (lactic acid) microspheres for drug delivery systems. Yonsei Med. J. 2000, 41, 720-734. [CrossRef] [PubMed]

5. Yang, F.; Murugan, R.; Ramakrishna, S.; Wang, X.; Ma, Y.X.; Wang, S. Fabrication of nano-structured porous PLLA scaffold intended for nerve tissue engineering. Biomaterials 2004, 25, 1891-1900. [CrossRef] [PubMed]

6. Ribeiro, C.; Sencadas, V.; Correia, D.M.; Lanceros-Méndez, S. Piezoelectric polymers as biomaterials for tissue engineering applications. Colloids Surf. B Biointerfaces 2015, 136, 46-55. [CrossRef] [PubMed]

7. Correia, D.M.; Sencadas, V.; Ribeiro, C.; Martins, P.M.; Martins, P.; Gama, F.M.; Botelho, G.; Lanceros-Méndez, S. Processing and size range separation of pristine and magnetic poly(l-lactic acid) based microspheres for biomedical applications. J. Colloid Interface Sci. 2016, 476, 79-86. [CrossRef] [PubMed]

8. Curry, E.J.; Ke, K.; Chorsi, M.T.; Wrobel, K.S.; Miller, A.N.; Patel, A.; Kim, I.; Feng, J.; Yue, L.; Wu, Q.; et al. Biodegradable Piezoelectric Force Sensor. Proc. Natl. Acad. Sci. USA 2018, 115, 909. [CrossRef]

9. Tajitsu, Y. Fundamental study on improvement of piezoelectricity of poly(t-lactic acid) and its application to film actuators. IEEE Trans. Ultrason. Ferroelectr. Freq. Control 2013, 60, 1625-1629. [CrossRef]

10. Noshadi, I.; Walker, B.W.; Portillo-Lara, R.; Shirzaei Sani, E.; Gomes, N.; Aziziyan, M.R.; Annabi, N. Engineering Biodegradable and Biocompatible Bio-ionic Liquid Conjugated Hydrogels with Tunable Conductivity and Mechanical Properties. Sci. Rep. 2017, 7, 4345. [CrossRef]

11. Ye, Y.-S.; Rick, J.; Hwang, B.-J. Ionic liquid polymer electrolytes. J. Mater. Chem. A 2013, 1, $2719-2743$. [CrossRef]

12. Dong, K.; Liu, X.; Dong, H.; Zhang, X.; Zhang, S. Multiscale Studies on Ionic Liquids. Chem. Rev. 2017, 117, 6636-6695. [CrossRef] [PubMed]

13. Zhang, S.G.; Zhang, Q.H.; Zhang, Y.; Chen, Z.J.; Watanabe, M.; Deng, Y.Q. Beyond solvents and electrolytes: Ionic liquids-based advanced functional materials. Prog. Mater. Sci. 2016, 77, 80-124. [CrossRef]

14. Lei, Z.; Chen, B.; Koo, Y.-M.; MacFarlane, D.R. Introduction: Ionic Liquids. Chem. Rev. 2017, 117, $6633-6635$. [CrossRef] [PubMed]

15. Xu, P.; Cui, Z.-P.; Ruan, G.; Ding, Y.-S. Enhanced Crystallization Kinetics of PLLA by Ethoxycarbonyl Ionic Liquid Modified Graphene. Chin. J. Polym. Sci. 2019, 37, 243-252. [CrossRef]

16. Correia, D.M.; Fernandes, L.C.; Martins, P.M.; García-Astrain, C.; Costa, C.M.; Reguera, J.; Lanceros-Méndez, S. Ionic Liquid-Polymer Composites: A New Platform for Multifunctional Applications. Adv. Funct. Mater. 2020, 1909736. [CrossRef]

17. Yoo, C.G.; Pu, Y.Q.; Ragauskas, A.J. Ionic liquids: Promising green solvents for lignocellulosic biomass utilization. Curr. Opin. Green Sustain. Chem. 2017, 5, 5-11. [CrossRef]

18. Marr, P.C.; Marr, A.C. Ionic liquid gel materials: applications in green and sustainable chemistry. Green Chem. 2016, 18, 105-128. [CrossRef]

19. Mejri, R.; Dias, J.C.; Hentati, S.B.; Botelho, G.; Esperanca, J.; Costa, C.M.; Lanceros-Mendez, S. Imidazolium-based ionic liquid type dependence of the bending response of polymer actuators. Eur. Polym. J. 2016, 85, 445-451. [CrossRef] 
20. Dias, J.C.; Correia, D.M.; Costa, C.M.; Ribeiro, C.; Maceiras, A.; Vilas, J.L.; Botelho, G.; de Zea Bermudez, V.; Lanceros-Mendez, S. Improved response of ionic liquid-based bending actuators by tailored interaction with the polar fluorinated polymer matrix. Electrochim. Acta 2019, 296, 598-607. [CrossRef]

21. Terasawa, N. High-performance transparent actuator made from Poly(dimethylsiloxane)/Ionic liquid gel. Sens. Actuators B-Chem. 2018, 257, 815-819. [CrossRef]

22. Wei, T.; Pang, S.; Xu, N.; Pan, L.; Zhang, Z.; Xu, R.; Ma, N.; Lin, Q. Crystallization behavior and isothermal crystallization kinetics of PLLA blended with ionic liquid, 1-butyl-3-methylimidazolium dibutylphosphate. J. Appl. Polym. Sci. 2015, 132, 41308. [CrossRef]

23. Gui, H.; Li, Y.; Chen, S.; Xu, P.; Zheng, B.; Ding, Y. Effects of biodegradable imidazolium-based ionic liquid with ester group on the structure and properties of PLLA. Macromol. Res. 2014, 22, 583-591. [CrossRef]

24. Gardella, L.; Furfaro, D.; Galimberti, M.; Monticelli, O. On the development of a facile approach based on the use of ionic liquids: preparation of PLLA (sc-PLA)/high surface area nano-graphite systems. Green Chem. 2015, 17, 4082-4088. [CrossRef]

25. Correia, D.M.; Barbosa, J.C.; Costa, C.M.; Reis, P.M.; Esperança, J.M.S.S.; de Zea Bermudez, V.; Lanceros-Méndez, S. Ionic Liquid Cation Size-Dependent Electromechanical Response of Ionic Liquid/Poly(vinylidene fluoride)-Based Soft Actuators. J. Phys. Chem. C 2019, 123, 12744-12752. [CrossRef]

26. Ribeiro, C.; Sencadas, V.; Costa, C.M.; Gómez Ribelles, J.L.; Lanceros-Méndez, S. Tailoring the morphology and crystallinity of poly(L-lactide acid) electrospun membranes. Sci. Technol. Adv. Mater. 2011, 12, 015001. [CrossRef]

27. Mazumder, M.; Ahmed, R.; Wajahat Ali, A.; Lee, S.-J. SEM and ESEM techniques used for analysis of asphalt binder and mixture: A state of the art review. Constr. Build. Mater. 2018, 186, 313-329. [CrossRef]

28. Ribeiro, C.; Costa, C.M.; Correia, D.M.; Nunes-Pereira, J.; Oliveira, J.; Martins, P.; Gonçalves, R.; Cardoso, V.F.; Lanceros-Méndez, S. Electroactive poly(vinylidene fluoride)-based structures for advanced applications. Nat. Protoc. 2018, 13, 681-704. [CrossRef]

29. Kawai, T.; Rahman, N.; Matsuba, G.; Nishida, K.; Kanaya, T.; Nakano, M.; Okamoto, H.; Kawada, J.; Usuki, A.; Honma, N.; et al. Crystallization and Melting Behavior of Poly (l-lactic Acid). Macromolecules 2007, 40, 9463-9469. [CrossRef]

30. Krikorian, V.; Pochan, D.J. Crystallization Behavior of Poly(l-lactic acid) Nanocomposites: Nucleation and Growth Probed by Infrared Spectroscopy. Macromolecules 2005, 38, 6520-6527. [CrossRef]

31. Cao, X.; Mohamed, A.; Gordon, S.H.; Willett, J.L.; Sessa, D.J. DSC study of biodegradable poly(lactic acid) and poly(hydroxy ester ether) blends. Thermochim. Acta 2003, 406, 115-127. [CrossRef]

32. Kiefer, J.; Fries, J.; Leipertz, A. Experimental Vibrational Study of Imidazolium-Based Ionic Liquids: Raman and Infrared Spectra of 1-Ethyl-3-methylimidazolium Bis(Trifluoromethylsulfonyl)imide and 1-Ethyl-3-methylimidazolium Ethylsulfate. Appl. Spectrosc. 2007, 61, 1306-1311. [CrossRef]

33. Jiao, Q.; Chen, Q.; Wang, L.; Chen, H.L.; Li, Y.J. Investigation on the Crystallization Behaviors of Polyoxymethylene with a Small Amount of Ionic Liquid. Nanomaterials 2019, 9. [CrossRef] [PubMed]

34. Correia, D.M.; Costa, C.M.; Lizundia, E.; Sabater i Serra, R.; Gómez-Tejedor, J.A.; Biosca, L.T.; Meseguer-Dueñas, J.M.; Gomez Ribelles, J.L.; Lanceros-Méndez, S. Influence of Cation and Anion Type on the Formation of the Electroactive $\beta$-Phase and Thermal and Dynamic Mechanical Properties of Poly(vinylidene fluoride)/Ionic Liquids Blends. J. Phys. Chem. C 2019, 123, 27917-27926. [CrossRef]

35. Mirkhalaf, S.M.; Fagerström, M. The mechanical behavior of polylactic acid (PLA) films: fabrication, experiments and modelling. Mech. Time-Depend. Mater. 2019. [CrossRef]

36. Aziz, S.B.; Woo, T.J.; Kadir, M.F.Z.; Ahmed, H.M. A conceptual review on polymer electrolytes and ion transport models. J. Sci. Adv. Mater. Devices 2018, 3, 1-17. [CrossRef]

37. Reizabal, A.; Correia, D.M.; Costa, C.M.; Perez-Alvarez, L.; Vilas-Vilela, J.L.; Lanceros-Méndez, S. Silk Fibroin Bending Actuators as an Approach Toward Natural Polymer Based Active Materials. ACS Appl. Mater. Interfaces 2019, 11, 30197-30206. [CrossRef]

(C) 2020 by the authors. Licensee MDPI, Basel, Switzerland. This article is an open access article distributed under the terms and conditions of the Creative Commons Attribution (CC BY) license (http://creativecommons.org/licenses/by/4.0/). 